\title{
Hepatic profile analyses of tipranavir in Phase II and III clinical trials Jaromir Mikl*1,2, Mark S Sulkowski ${ }^{3}$, Yves Benhamou ${ }^{4}$, Douglas Dieterich ${ }^{5}$, Stanislas Pol ${ }^{6}$, Jürgen Rockstroh ${ }^{7}$, Patrick A Robinson ${ }^{2}$, Mithun Ranga ${ }^{2}$ and Jerry O Stern ${ }^{2}$
}

\author{
Address: ${ }^{1}$ SUNY at Albany, School of Public Health, Rensselaer NY, USA, ${ }^{2}$ Boehringer Ingelheim Pharmaceuticals, Inc., Ridgefield, CT, USA \\ ${ }^{3}$ Division of Infectious Diseases, Johns Hopkins University School of Medicine, Baltimore, USA, ${ }^{4}$ Service d'Hépato-Gastroentérologie, Hôpital \\ Pitié-Salpêtrière, Paris, France, ${ }^{5}$ Mount Sinai School of Medicine, New York, New York, USA, ${ }^{6}$ Hôpital Necker, APHP, Unité d'Hépatologie, Paris, \\ France and ${ }^{7}$ Department of Medicine I, University of Bonn, Bonn, Germany \\ Email: Jaromir Mikl* - jmikl1@nycap.rr.com; Mark S Sulkowski - msulkowski@jhmi.edu; Yves Benhamou - ybenhamou@teaser.fr; \\ Douglas Dieterich - douglas.dieterich@msnyuhealth.org; Stanislas Pol - stanislas.pol@cch.aphp.fr; \\ Jürgen Rockstroh - Juergen.Rockstroh@ukb.uni-bonn.de; Patrick A Robinson - patrick.robinson@boehringer-ingelheim.com; \\ Mithun Ranga - mithun.ranga@boehringer-ingelheim.com; Jerry O Stern - jerry.stern@boehringer-ingelheim.com \\ * Corresponding author
}

Published: 14 December 2009

BMC Infectious Diseases 2009, 9:203 doi:10.1 186/147/-2334-9-203
Received: 15 April 2009

Accepted: 14 December 2009

This article is available from: http://www.biomedcentral.com//47/-2334/9/203

(c) 2009 Mikl et al; licensee BioMed Central Ltd.

This is an Open Access article distributed under the terms of the Creative Commons Attribution License (http://creativecommons.org/licenses/by/2.0), which permits unrestricted use, distribution, and reproduction in any medium, provided the original work is properly cited.

\begin{abstract}
Background: The risk and course of serum transaminase elevations (TEs) and clinical hepatic serious adverse event (SAE) development in ritonavir-boosted tipranavir (TPV/r) 500/200 mg BID recipients, who also received additional combination antiretroviral treatment agents in clinical trials (TPV/r-based CART), was determined.

Methods: Aggregated transaminase and hepatic SAE data through 96 weeks of TPV/r-based cART from five Phase Ilb/lll trials were analyzed. Patients were categorized by the presence or absence of underlying liver disease (+LD or -LD). Kaplan-Meier (K-M) probability estimates for time-to-first US National Institutes of Health, Division of AIDS (DAIDS) Grade 3/4 TE and clinical hepatic SAE were determined and clinical actions/outcomes evaluated. Risk factors for DAIDS Grade 3/4 TE were identified through multivariate Cox regression statistical modeling.

Results: Grade 3/4 TEs occurred in I44/I299 (II.1\%) patients; I23/I44 (85\%) of these were asymptomatic; $84 \%$ of these patients only temporarily interrupted treatment or continued, with transaminase levels returning to Grade $\leq 2$. At 96 weeks of study treatment, the incidence of Grade $3 / 4$ TEs was higher among the +LD (16.8\%) than among the -LD (I0.1\%) patients. K-M analysis revealed an incremental risk for developing DAIDS Grade 3/4 TEs; risk was greatest through 24 weeks $(6.1 \%)$, and decreasing thereafter (>24-48 weeks: $3.4 \%,>48$ weeks-72 weeks: $2.0 \%,>72-96$ weeks: $2.2 \%$ ), and higher in +LD than -LD patients at each 24-week interval. Treatment with TPV/ $r$, co-infection with hepatitis B and/or C, DAIDS grade >I TE and CD4 $4^{+}>200$ cells $/ \mathrm{mm}^{3}$ at baseline were found to be independent risk factors for development of DAIDS Grade 3/4 TE; the hazard ratios (HR) were 2.8, 2.0, 2.I and I.5, respectively. Four of the I44 (2.7\%) patients with Grade 3/ 4 TEs developed hepatic SAEs; overall, I4/I 299 (I.I\%) patients had hepatic SAEs including six with hepatic failure (0.5\%). The K-M risk of developing hepatic SAEs through 96 weeks was I.4\%; highest risk was observed during the first 24 weeks and decreased thereafter; the risk was similar between
\end{abstract}


+LD and -LD patients for the first 24 weeks $(0.6 \%$ and $0.5 \%$, respectively) and was higher for +LD patients, thereafter.

Conclusion: Through 96 weeks of TPV/r-based cART, DAIDS Grade 3/4 TEs and hepatic SAEs occurred in approximately $\mathrm{I} I \%$ and I\% of TPV/r patients, respectively; most (84\%) had no significant clinical implications and were managed without permanent treatment discontinuation. Among the 14 patients with hepatic SAE, 6 experienced hepatic failure $(0.5 \%)$; these patients had profound immunosuppression and the rate appears higher among hepatitis co-infected patients. The overall probability of experiencing a hepatic SAE in this patient cohort was $1.4 \%$ through 96 weeks of treatment. Independent risk factors for DAIDS Grade 3/4 TEs include TPV/r treatment, co-infection with hepatitis B and/or C, DAIDS grade >I TE and CD4 $>200$ cells $/ \mathrm{mm}^{3}$ at baseline.

Trial registration: US-NIH Trial registration number: NCT00 I44 I 70

\section{Background}

The introduction of highly active antiretroviral therapy (HAART) is responsible for a substantial decline in AIDS progression, opportunistic infections and mortality in HIV-1-infected individuals in recent years [1-3]. However, in the presence of antiretroviral (ARV) drugs and nonadherence, resistant strains emerge and contribute to therapeutic failure [4]. Tipranavir (TPV; Aptivus ${ }^{\circledR}$ is a next-generation protease inhibitor (PI), used in combination with low-dose ritonavir (RTV;/r) in combination with other background antiretroviral agents (TPV/r-based cART) for treatment-experienced, HIV-1 infected patients with resistance to more than one PI. TPV/r has shown sustained activity against viruses with up to 20 PI mutations [5]. However, TPV/r-based cART use is associated with a risk of elevated serum levels of alanine aminotransferase (ALT) and/or aspartate aminotransferase (AST) transaminases [6].

In general, HAART may be associated with elevated serum transaminases [7]. The reported incidence of severe transaminase elevations associated with HAART varies from a low of $2 \%$ to as high as $18 \%$ [8]. However, reports from other investigators indicate that as many as 6\%-30\% of ARV treated patients, particularly those receiving protease inhibitors, may experience significant increases in serum liver enzymes and consequently may have to discontinue treatment $[9,10]$. Patients co-infected with viral hepatitis are at increased risk for hepatotoxicity [8,11-13]. Considering that between $10 \%-50 \%$ of HIV infected persons in the US and Europe are estimated to be co-infected with HCV [14-17] hepatotoxicity becomes a challenge when treating these patients. Severe hepatotoxicity occurs in up to $6 \%$ of patients on HAART [7].

A recent study evaluated patients receiving PI-based ARV therapy, with or without RTV co-administration [9]; the risk of developing hepatotoxicity was similar across the PIs evaluated and a boosting dose of RTV was associated with a much lower risk than was observed with therapeu- tic RTV dosing [9]. The study concluded that, while druginduced liver toxicity was an important consideration for HIV treatment decisions, potential efficacy and pre-existing resistance primarily influence the selection of a cART regimen [9]. In a recent report, the Kaplan-Meier (K-M) risk estimates of US National Institutes of Health, Division of AIDS (DAIDS) [18] Grade 3/4 ALT elevations were $9.9 \%$ and $2.8 \%$, respectively, in patients treated with TPV/ $\mathrm{r}$ or RTV-boosted comparator PIs $(\mathrm{CPI} / \mathrm{r})$ in the large Phase III RESIST trials at 48 weeks [19]. Multivariate analyses in TPV/r-based cART patients indicated that the most significant risk factors for Grade 3/4 ALT/AST levels in the $\mathrm{TPV} / \mathrm{r}$ and CPI/r groups were elevated baseline transaminases and hepatitis co-infection [19]. Others observed similar results, identifying elevated transaminases at baseline and/or hepatitis co-infection as risk factors associated with liver toxicity among HIV infected patients treated with antiretrovirals [20-24].

The current analysis is based largely on patients enrolled in the RESIST trial, which demonstrated superior TPV/r efficacy and durability in suppressing viral load (VL), compared to $\mathrm{CPI} / \mathrm{r}$ in more than 1400 patients with PI resistance, advanced HIV infection and few, if any, other treatment options [6].

The objective of this hepatic safety analysis was to evaluate the risk and natural history of serum ALT/AST transaminase elevations and clinical hepatic serious adverse event (SAE) development among treatment-experienced patients receiving the approved standard dose of 500/200 $\mathrm{mg}$ BID TPV/r in Phase IIb and III trials through 96 weeks of treatment. The development of DAIDS Grade 3/4 ALT/ AST elevations was further evaluated in patients categorized as having underlying liver disease (+LD; baseline transaminase elevations DAIDS Grade >1 [18] and/or hepatitis $\mathrm{B} / \mathrm{C}$ virus [HBV/HCV] co-infection) or no underlying liver disease (-LD; baseline transaminase elevations Grade $\leq 1$ and no HBV/HCV co-infection). 


\section{Methods}

Aggregated safety data were pooled from the five Phase IIb and III trials which comprised the clinical development program for TPV/r. Patients in these trials were highly treatment-experienced and received the approved dose of $\mathrm{TPV} / \mathrm{r} 500 / 200 \mathrm{mg}$ BID. The five trials in which these patients were enrolled were two Phase II trials (1182.51 $[\mathrm{N}=67], 1182.52[\mathrm{n}=72])[25,26]$, two pivotal Phase III trials (1182.12 [RESIST $1, \mathrm{n}=311$ ], 1182.48 [RESIST 2, $\mathrm{n}$ $=436])[6,27,28]$ and one "roll-over" trial $(1182.17[\mathrm{n}=$ 413]) [29] that allowed patients from completed Phase II trials to continue to receive TPV/r 500/200 mg BID and provided TPV/r to those in RESIST who failed on the CPI/ $\mathrm{r}$ therapy. The RESIST-1 and RESIST-2 trials were openlabel, randomized trials that compared TPV/r with $\mathrm{CPI} / \mathrm{r}$ regimens in 21 countries in Europe, North and South America, and Australia $[6,27,28]$. Trial 1182.52 was a randomized, dose-selection trial thatcompared three dose regimens of TPV/r, including the dose of 500/200 mg BID [25]. Trial 1182.51 was a randomizedpharmacokinetic and safety comparison of TPV/r versus CPI/r for 2 weeks, followed by a dual-boosted regimen in patients who were ineligible for the pivotal Phase III trials because of resistance requirements [26].

All patients included in these five trials were HIV-1infected male and female adults (aged $\geq 18$ years) with $\geq$ 3 months of prior treatment with three classes of ARVs (nucleoside reverse transcriptase inhibitors [NRTIs], nonnucleoside reverse transcriptase inhibitors [NNRTIs], and PIs), including at least two prior PI-based regimens (one being the regimen at screening), who had a VL of $\geq 1000$ copies/mL of plasma HIV-1 RNA at baseline. Patients with $\mathrm{HBV}$ or HCV were eligible for inclusion if serum ALT/AST transaminase levels were DAIDS Grade $2(\leq 2.5 \times$ the upper limit of normal [ULN] reference range) or less [18]. Patients who were failing CPI/ $\mathrm{r}$ in the RESIST trials or who were treated with TPV/ $\mathrm{r}$ in Phase II trials were allowed to participate in the open-label TPV/r roll-over study (1182.17) and were included in the hepatic safety analyses.

All studies were designed and monitored in accordance with the International Conference on Harmonization guidance for Good Clinical Practice (ICH GCP) and written informed consent was obtained from all study participants. All study protocols, informed consent forms and trial documentation was reviewed and approved by the institutional review boards/local ethics committees of participating centers.

The course of transaminase elevations and hepatic SAEs through 96 weeks was characterized. Transaminase elevations were categorized according to NIH, DAIDS [18] system for Grading the Severity of Adult and Pediatric Adverse Events based on multiples of the Upper Limit of
Normal (ULN) reference range for ALT/AST: DAIDS Grade 0 defined as ALT/AST within normal range through $<1.25$ $\times$ ULN; Grade 1 defined as mild elevation (1.25-2.5 × ULN); DAIDS Grade 2 defined as moderate elevation $(>2.5-5.0 \times$ ULN); DAIDS Grade 3 defined as ALT/AST $>$ $5-10 \times$ ULN; and DAIDS Grade 4 defined as ALT/AST $>10$ $\times$ ULN. K-M probability estimates for time-to-first DAIDS Grade 3/4 ALT/AST elevation and time-to-first clinical hepatobiliary SAE were determined. Clinical actions and outcomes following the development of DAIDS Grade 3/ 4 transaminase elevation were analyzed with regards to treatment continuation, interruption or discontinuation of TPV/r.

Potential risk factors for DAIDS Grade 3/4 for ALT and/or AST were assessed through multivariate Cox regression statistical modeling and included all RESIST 1 and 2 patients $(\mathrm{N}=1486)$ in order to compare TPV/r and $\mathrm{CPI} / \mathrm{r}$ treated patients.

Hepatobiliary adverse events (AEs) were defined as any AE with a MedDRA (ver. 8.1) preferred term falling into hepatobiliary system organ class (SOC) and included serious and non-serious hepatobiliary AEs regardless of severity. All of these events were considered to represent "symptomatic" AEs. Complete clinical profiles (all laboratory and reported AEs from baseline throughout the participation of the patient in the study) were created and underwent medical review. Events were excluded as representing symptomatic "hepatic" events if they were due to biliary disease alone (e.g. cholecystitis, gallbladder cyst, choledocholithiasis), hepatic neoplasm alone (primary or secondary), or if they represented only asymptomatic elevations of liver laboratory tests, with the absence of any other associated clinical AEs (e.g., fever, malaise, nausea, abdominal pain, etc).

Patients were classified as $+\mathrm{LD}$ retrospectively by the presence of either chronic viral hepatitis and/or elevated serum transaminases DAIDS Grade $>1$ at baseline [19]. Study protocols excluded patients with screening ALT/AST elevation of Grade $\geq 2$; however, some patients $(n=48)$ who met the screening criteria were found to have DAIDS Grade 2 elevations at baseline on the first study visit and were included in the trials. Chronic HCV infection was defined by the detection of HCV RNA. Chronic HBV infection was assessed at baseline by the presence of hepatitis B surface antigen (HBV DNA was not measured). Patients who were not co-infected with HBV or HCV and who had baseline transaminase elevations of Grade 1 or less were classified as -LD for these analyses.

\section{Results \\ Baseline characteristics}

Baseline characteristics of all study patients are presented in Table 1. Data aggregated from the five TPV/r trials iden- 
tified 1299 treatment-experienced patients who received the approved 500/200 mg BID dose of TPV/r. Of these, 1088 were classified as $-\mathrm{LD}$ patients and 179 as $+\mathrm{LD}$ patients. The remaining 32 patients had either HBV/HCV co-infection status or transaminase elevation data missing at baseline and therefore are listed as unclassified and were excluded from the +LD and -LD sub-analyses.

The patients were mostly males $(86.5 \%)$ and there was similar gender distribution between the risk groups (Table 1). The median baseline HIV-1 RNA and CD4+ cell counts were similar between the two risk groups; -LD patients had a median HIV-1 RNA level of $4.8 \log _{10}$ copies/mL and a median CD4+ cell count of 158 cells $/ \mathrm{mm}^{3}$ at baseline, +LD patients had a median HIV RNA of $4.7 \log _{10}$ copies/ $\mathrm{mL}$ and a median CD4+ cell count of 151 cells $/ \mathrm{mm}^{3}$ at baseline.

Compared to patients who did not experience hepatic SAEs, the 14 patients who experienced hepatic SAE were slightly older, and presented with higher HIV RNA counts and lower $\mathrm{CD}^{+}{ }^{+}$counts at baseline (Table 1). All but one of these patients responded to TPV/ $\mathrm{r}$ treatment: the median maximal reduction from baseline in HIV RNA count was $5.3 \log _{10}$ and the median maximal CD4+ count increase from baseline was 74.5 cells $/ \mathrm{mm}^{3}$ (data not shown).

DAIDS Grade 3/4 ALT andlor AST transaminase elevations In the aggregate Phase II and III TPV/r trials $(\mathrm{n}=1299)$, $11.1 \%$ of TPV/r patients receiving 500/200 mg BID developed DAIDS Grade 3/4 ALT/AST elevations through 96 weeks of TPV/r-based cART treatment in the studies. The incidence of DAIDS Grade 3/4 events was 11.1\% (144/ 1299 subjects); of these $6.9 \%$ were Grade 3 and $4.2 \%$ were Grade 4 elevations.

Cumulative K-M estimates through 96 weeks of study treatment for time-to-first DAIDS Grade 3/4 ALT/AST elevations are presented in Table 2 and Figure 1 . In the overall patient population, the cumulative risk of developing a DAIDS Grade 3/4 ALT/AST elevation while on study treatment was greatest in the first 24 weeks $(6.1 \%)$ compared to subsequent 24 week periods. The subsequent

Table I: Demographic and baseline characteristics of TPV/r 500/200 mg patients in trials I I82.12, I I82.48, I I82.5 I, I I82.52 and I I82.I7 by risk

\begin{tabular}{|c|c|c|c|c|}
\hline & $\begin{array}{l}\text { All TPV/rI } \\
N=1299\end{array}$ & $\begin{array}{c}\text { TPV/r }- \text { LD }^{2} \\
N=1088\end{array}$ & $\begin{array}{c}\text { TPV/r +LD } \\
N=179\end{array}$ & $\begin{array}{c}\text { TPV/r SAE } \\
N=14\end{array}$ \\
\hline \multicolumn{5}{|l|}{ Age (years): } \\
\hline Median & 43 & 43 & 42 & 42 \\
\hline Range & $17-80$ & $17-80$ & $18-72$ & $35-63$ \\
\hline \multicolumn{5}{|l|}{ Gender [N (\%)]: } \\
\hline Male & II $24(86.5)$ & $939(86.3)$ & $160(89.4)$ & $13(92.9)$ \\
\hline Female & $175(13.5)$ & 149 (13.7) & $19(10.6)$ & I (7.1) \\
\hline \multicolumn{5}{|l|}{ Baseline HIV RNA ( $\log _{10}$ copies $\left./ \mathrm{mL}\right)$ : } \\
\hline Median & 4.8 & 4.8 & 4.7 & 4.9 \\
\hline Range & 1.7-6.5 & $1.7-6.5$ & $2.7-6.3$ & $3.6-5.6$ \\
\hline \multicolumn{5}{|l|}{ Baseline CD4+ cell count (cells $\left./ \mathrm{mm}^{3}\right)$ : } \\
\hline Median & 156 & 158 & 151 & 70 \\
\hline Range & $1-1893$ & $1-1893$ & $1-702$ & $4-337$ \\
\hline \multicolumn{5}{|l|}{ Hepatitis co-infection [N (\%)]: } \\
\hline $\mathrm{HBsAg-/HCV} \mathrm{RNA-}$ & $1136(87.5)$ & $1088(100.0)$ & $37(20.7)$ & $9(64.3)$ \\
\hline $\mathrm{HBsAg+}$ & $55(4.2)$ & $0(0.0)$ & $55(30.7)$ & $2(14.3)$ \\
\hline HCV RNA+ & $83(6.4)$ & $0(0.0)$ & $83(46.4)$ & $3(21.4)$ \\
\hline $\mathrm{HBsAg}+/ \mathrm{HCV}$ RNA+ & $2(0.2)$ & $0(0.0)$ & $2(1.1)^{\prime}$ & $0(0.0)$ \\
\hline missing & $23(1.8)$ & $0(0.0)$ & $2(1.1)$ & $0(0.0)$ \\
\hline Baseline DAIDS Grade $\geq 2$ ALT/AST ${ }^{3}$ & $51(3.9)$ & $0(0.0)$ & $51(28.5)$ & $0(0.0)$ \\
\hline
\end{tabular}

IThirty-two patients had missing information and were unable to be classified into one of the two risk groups. These patients were more likely to be female $(21.9 \%)$ with lower CD4+ counts at baseline (median I2I cells $/ \mathrm{mm}^{3}$ ).

2+LD: patients with underlying liver disease (baseline evidence of active HBV/HCV infection or ALT/AST Grade > I); -LD: patients with no apparent liver disease (absence of active HBV/HCV infection and ALT/AST Grade $\leq 1$ ).

${ }^{3}$ At baseline, 48 patients had DAIDS Grade 2 ALT/AST, while three patients had DAIDS Grade 3 ALT/AST just prior to first dose of TPV/r. $\mathrm{ALT}=$ alanine aminotransferase; $\mathrm{AST}=$ aspartate aminotransferase; DAIDS = Division of AIDS; $\mathrm{HBsAg}=$ hepatitis $\mathrm{B}$ surface antigen; $\mathrm{HBV}=$ hepatitis $B$ virus; $\mathrm{HCV}=$ hepatitis $C$ virus; $T P V / r=$ ritonavir-boosted tipranavir 500/200 mg BID 
Table 2: Kaplan-Meier estimates for time-to-first DAIDS Grade 3/4 ALT and/or AST elevation and time-to-first hepatic serious adverse event (SAE) among TPV/r 500/200 mg patients in trials II82.1 2, II82.48, II82.5 I, II82.52 and II82.17

\begin{tabular}{|c|c|c|c|c|c|c|}
\hline \multirow[t]{2}{*}{96 week CR(\%)! } & \multicolumn{3}{|c|}{ DAIDS² Grade 3/4 ALT/AST } & \multicolumn{3}{|c|}{ Hepatic SAE } \\
\hline & $\begin{array}{c}\text { TPV/r } \\
\text { All } \\
\mathbf{N}=\text { I } 299\end{array}$ & $\begin{array}{c}\text { TPV/r-LD } \\
N=1088\end{array}$ & $\begin{array}{c}\text { TPV/r +LD } \\
\mathbf{N}=179\end{array}$ & $\begin{array}{c}\text { TPV/r } \\
\text { All } \\
\mathbf{N}=1299\end{array}$ & $\begin{array}{c}\text { TPV/r-LD } \\
\mathbf{N}=1088\end{array}$ & $\begin{array}{c}\text { TPV/r+LD } \\
N=179\end{array}$ \\
\hline Week 24 & 6.1 & 5.4 & 9.4 & 0.5 & 0.5 & 0.6 \\
\hline Week 48 & 9.5 & 8.3 & 15.6 & 0.9 & 0.7 & 2.1 \\
\hline Week 72 & 11.5 & 10.3 & 17.2 & 1.1 & 0.8 & 3.0 \\
\hline Week 96 & 13.7 & 12.5 & 20.6 & $\mathrm{I} .4$ & 1.0 & 4.3 \\
\hline
\end{tabular}

'Cumulative rate through 96 weeks.

2DAIDS Grade 3 is defined as ALT/AST $=5.1$ to $10 \times$ ULN reference range. DAIDS Grade 4 is defined as ALT/AST $>10 \times$ ULN reference range.

${ }^{3}$ Log-rank test comparing -LD versus +LD patients: $p<0.05$.

${ }^{4}$ Log-rank test comparing -LD versus +LD patients: $p<0.05$.

$+\mathrm{LD}=$ patients with underlying liver disease (baseline evidence of active HBV/HCV infection or ALT/AST Grade >I); -LD = patients with no apparent liver disease (absence of active HBV/HCV infection and ALT/AST Grade $\leq$ I); ALT = alanine aminotransferase; AST = aspartate aminotransferase; DAIDS = Division of AIDS; HBV = hepatitis $B$ virus; $\mathrm{HCV}=$ hepatitis $C$ virus; $\mathrm{SAE}=$ serious adverse event; $T P V / r=$ ritonavir-

boosted tipranavir; ULN = upper limit of normal

cumulative risk estimate was $9.5 \%$ at 48 weeks (an accumulation of $3.4 \%$ additional risk in the period from 24 to 48 weeks). At 72 weeks the cumulative risk was $11.5 \%$ (additional 2.0\% in the period from 48 weeks to 72 weeks) and at 96 weeks the cumulative risk was $13.7 \%$ (an additional $2.2 \%$, for 72 to 96 weeks). The proportion of patients with DAIDS Grade 3/4 transaminase elevations by Week 96 was lower among -LD patients (10.1\%; 110/ $1088)$, compared to $+\mathrm{LD}$ patients $(16.8 \% ; 30 / 179)$. The K-M cumulative risk for Grade 3/4 ALT/AST elevations was nearly 2 -fold higher in $+\mathrm{LD}$ patients compared to their -LD counterparts at each 24 week interval (Table 2).

\section{Risk factors for DAIDS Grade 3/4 TE}

The RESIST 1 and 2 trials were randomized controlled trials that compared responses between TPV/r- based and comparator PI/r- (CPI/r-) based cART. The design and the patient population have been described previously $[19,27,28,30,31]$. To further understand the association of risk factors with the development of severe liver transaminase elevations, Cox regression model was constructed. This model examined time to first DAIDS Grade 3/4 ALT and/or AST elevations in RESIST patients, using $96+$ week data. Risk factors found to be associated with time to first Grade 3/4 ALT and/or AST elevations while controlling for other factors in the model (see Table 3 ) included TPV/r treatment (HR: 2.8; $\mathrm{p}<0.05$ ), HBV/HCV co-infection (HR: 2.0; $\mathrm{p}<0.05$ ), elevated (DAIDS Grade $\geq 2$ ) ALT and/or AST at baseline (HR: 2.1; $<<0.10$ ), and baseline CD4 > 200 cells/mm $\mathrm{mm}^{3}$ (HR: 1.5; p < 0.10).
Two additional Cox models examined only the TPV/r patients in RESIST 1 and 2, and compared the risk of DAIDS Grade 3/4 ALT and/or AST elevation in patients who were either HBV/HCV co-infected or who had DAIDS Grade $\geq 2$ for ALT and/or AST at baseline versus those patients who had neither co-infection nor baseline ALT and/or AST elevation. The analyses used 96+ week data. In both comparisons, the risk was significantly elevated (HR: $1.8, \mathrm{p}<0.05)$ in patients who had co-infection or $\geq 2$ DAIDS Grade ALT and/or AST at baseline (Data not shown).

\section{Clinical outcomes following Grade 3/4 ALTIAST transaminase elevations}

Of the 144 patients who developed DAIDS Grade 3/4 ALT/AST elevations by Week 96, there were 123 (85\%) who had no associated symptomatic hepatic events (asymptomatic). Over this period, 1.6\% (21/1299) of TPV/r 500/200 mg BID recipients developed DAIDS Grade 3/4 ALT/AST elevations, accompanied by symptomatic hepatic AEs (serious and/or non-serious) and 0.3\% $(\mathrm{n}=4)$ of all TPV/r patients developed a hepatic SAE in association with a DAIDS Grade 3/4 ALT/AST elevation.

The majority (84.0\%) of the 144 patients were either able to continue TPV/r treatment or re-introduce treatment following interruption (Figure 2).

Following the development of DAIDS Grade 3/4 ALT/AST elevations, $64.6 \%(93 / 144)$ of patients continued TPV/r treatment without interruption, and in $94.6 \%$ of these, 


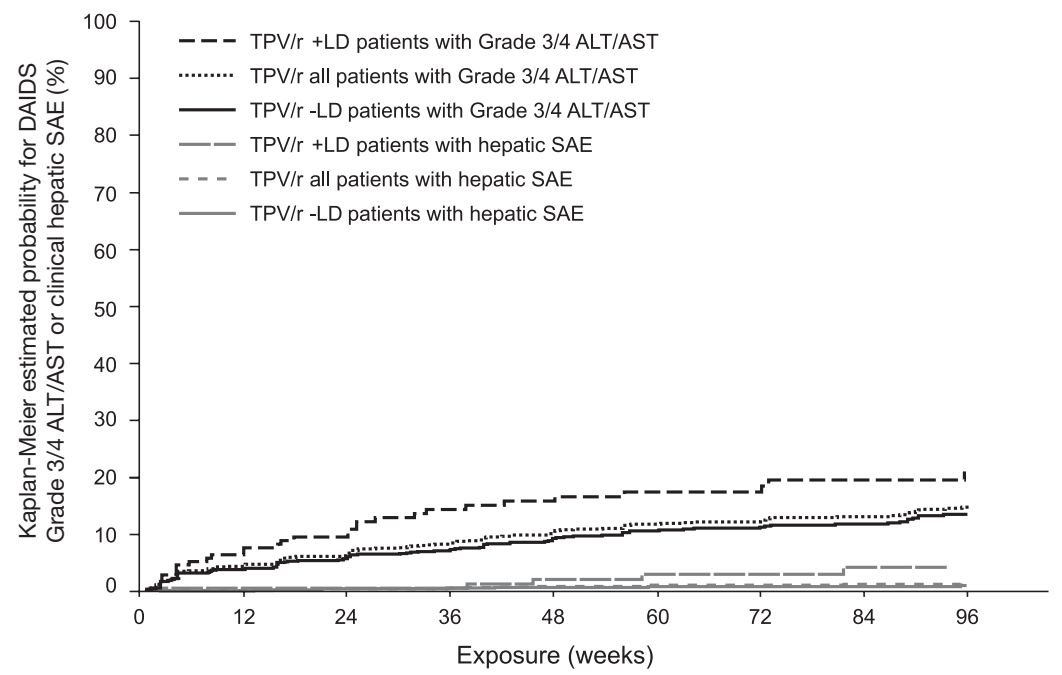

Figure I

Kaplan-Meier estimates for time-to-first Grade 3/4 ALTIAST elevations and to first hepatobiliary serious adverse events (SAEs) among TPV/r recipients by baseline risk group. Log-rank test $p$-value comparing -LD vs. +LD:. Incidence of Hepatic SAEs $p=0.0125$. Incidence of DAIDS Grade 3/4 ALT/AST $p=0.0072$. TPV/r +LD patients with Grade 3/ $4 \mathrm{ALT} / \mathrm{AST}=\mathrm{TPV} / \mathrm{r}$ patients co-infected with $\mathrm{HBV} / \mathrm{HCV}$ or with baseline ALT/AST DAIDS > I, time-to-first DAIDS 3 ALT/ AST. TPV/r -LD patients with Grade 3/4 ALT/AST = TPV/r patients not co-infected with HBV/HCV and with baseline ALT/AST DAIDS I, time-to-first DAIDS 3 ALT/AST. TPV/r +LD patients with hepatic SAE = TPV/r patients co-infected with HBV/HCV or with baseline ALT/AST DAIDS > I, time-to-first onset date of hepatic SAE. TPV/r -LD patients with hepatic SAE = TPV/r patients not co-infected with HBV/HCV and with baseline ALT/AST DAIDS I, time-to-first onset date of hepatic SAE. +LD = patients with underlying liver disease (baseline evidence of active HBV/HCV infection or ALT/AST DAIDS >I); -LD = patients with no apparent liver disease (absence of active HBV/HCV infection and ALT/AST Grade $\leq \mathrm{I}$ ); ALT = alanine aminotransferase; $\mathrm{AST}=$ aspartate aminotransferase; DAIDS = Division of AIDS; HBV = hepatitis B virus; HCV = hepatitis C virus; TPV/r = ritonavir-boosted tipranavir.

ALT/AST returned to Grade 2 or less in a median of 32 days (IQR $=14-84$ days), while remaining on treatment.

There were 28/144 (19.4\%) patients who had at least one treatment interruption. For 17/28 (60.7\%) patients who interrupted therapy, elevated transaminases returned to DAIDS Grade 2 or less after a median of 46 days (IQR = $20-58$ days). However, 11/28 (39.3\%) patients who had treatment interruption developed subsequent recurrence(s) of DAIDS Grade 3/4 transaminases that led to discontinuation.

Twenty of the 144 (13.9\%) patients with DAIDS Grade 3/ 4 ALT/AST elevations discontinued treatment at the first Grade 3/4 elevation. Three patients developed their first severe transaminase elevations on the last visit for which follow-up data were available.

The course of action taken among -LD and +LD TPV/r patients was similar (Figure 2), with the majority (81/110 [74\%] -LD patients; $22 / 30$ [73\%] +LD patients) able to continue, or interrupt and re-introduce, TPV/r treatment with a subsequent decrease in transaminase elevations to
DAIDS Grade 2 or less. All -LD patients who continued with TPV/r treatment had transaminase levels return to DAIDS Grade 2 or less after a median of 29 days. Similarly, among +LD patients who continued with TPV/r treatment, all but one patient had transaminase levels decrease to DAIDS Grade 2 or less, although the median recovery time was longer than in -LD patients (43 days).

\section{Clinical hepatic serious adverse events}

Among the 1299 treatment-experienced TPV/r patients, $14(1.1 \%)$ patients experienced hepatic (non-biliary and non-laboratory) SAEs.

The cumulative risk of clinical hepatic SAEs remained low through 96 weeks $(0.5 \%$ at Week $24,0.9 \%$ at Week 48 , and $1.4 \%$ at Week 96) (Table 2 and Figure 1). Among -LD and $+\mathrm{LD}$ patients, the cumulative risks of hepatic SAEs were similar during the first 24 weeks $(0.5 \%$ and $0.6 \%$, respectively). However, after Week 24, the risks of clinical hepatic SAEs increased disproportionately among $+\mathrm{LD}$ patients compared to $-\mathrm{LD}$ patients. K-M rates of $+\mathrm{LD}$ patients (Figure 1) increased to $2.1 \%$ at Week 48 (an accumulation of $1.5 \%$ additional risk from Week 24 to Week 
Table 3: Cox regression for time to first occurrence of DAIDS Grade 3 or 4 ALT and/or AST abnormalities in TPV/r-based and comparator $\mathrm{PI} / \mathrm{r}$ - (CPI/r-) based CART RESIST patients

\begin{tabular}{llc}
\hline Parameter & HR' (p-value) & 95\% Confidence Interval \\
\hline $\begin{array}{l}\text { Treatment group: } \\
\text { TPV/r } \vee \text { CPI/r }\end{array}$ & $2.77(<0.000 I)$ & $1.71,4.48$ \\
\hline $\begin{array}{l}\text { HBV or HCV co-infection: } \\
\text { yes vs. no }\end{array}$ & $2.00(0.0057)$ & $1.22,3.27$ \\
\hline $\begin{array}{l}\text { ALT/AST at baseline: } \\
\text { Grade } \geq 2 \text { vs. } \leq \text { Grade I }\end{array}$ & $2.05(0.0709)$ & $0.94,4.48$ \\
\hline $\begin{array}{l}\text { CD4+ cells at baseline: } \\
>200 \text { vs. } \leq 200 \text { cells } / \mathrm{mm}^{3}\end{array}$ & $1.46(0.0643)$ & $0.98,2.18$ \\
\hline
\end{tabular}

I Hazard Ratio

Model further controlled for sex, race and concurrent use of T-20 (none were statistically significant).

48 ), to $3.0 \%$ at Week 72 (accumulated additional risk of $0.9 \%$ from Week 48 to 72 ), and to $4.3 \%$ at Week 96 (accumulated additional risk of $1.3 \%$ from Week 72 to 96 ). For -LD patients, the corresponding accumulation of additional risk was $0.2 \%$ from Week 24 to Week 48, 0.1\% from Week 48 to 72 , and $0.2 \%$ from Week 72 to 96 .

Among the +LD patients there were 5/179 (2.8\%) with hepatic SAEs; two with hepatic failure, one with hepatic steatosis, one with hepatocellular damage and one with isolated hyperbilirubinemia. All five were co-infected with HBV or HCV. There were 9/1088 -LD patients $(0.8 \%)$ with hepatic SAEs; four with hepatic failure, two with hepatotoxicity or toxic hepatitis, and one patient each with steatosis, cirrhosis, hepatosplenomegaly.

Among all 1299 TPV/r-treated patients, six (0.5\%) had a hepatic SAE denoting hepatic failure. Five had fatal outcomes. These events occurred in patients with concomitant end-stage AIDS events, including disseminated Mycobacterium avium intracellulare, visceral leishmaniasis, Burkitt's lymphoma, squamous carcinoma, sepsis and multi-organ failure. The sixth patient recovered rapidly and was discharged from the hospital after five days, suggesting the diagnosis may have not been entirely accurate. An apparent higher risk among hepatitis co-infected patients was observed; two of the six hepatic failure cases occurred among hepatitis co-infected patients $(1.4 \%)$ while four occurred among non-hepatitis co-infected patients $(0.4 \%$; data not shown). In all but one case, the extent of the role of TPV in the development of liver failure is uncertain.

The occurrence of Grade 3/4 ALT/AST elevations and clinical hepatic SAEs among TPV/r 500/200 mg BID patients who were - $\mathrm{LD}$ and $+\mathrm{LD}$ is presented in Table 4 . Of the 144 patients who developed Grade 3/4 ALT/AST elevations, four subsequently developed a hepatic SAE through 96 weeks, suggesting that transaminase elevations may be a poor predictor of clinical hepatic SAEs (positive predictive value [PPV]: $2.8 \%$ ). The $+\mathrm{LD}$ patients had a PPV of just $3.3 \%$, with only $1 / 30$ patients with Grade 3/4 ALT/AST elevations developing a hepatic SAE. The -LD patients had a PPV of $2.7 \%$, with $3 / 110$ patients with a Grade $3 / 4$ ALT/ AST elevation through 96 weeks developing a hepatic SAE.

Among -LD and +LD patients treated with TPV/r 500/200 $\mathrm{mg}$ BID in the five trials comprising the TPV/r development program, monitoring of transaminase elevations to determine which patients may be at risk for clinical hepatic SAEs appears to have limited value, with corresponding sensitivity values of $33.3 \%$ and $20.0 \%$. In both patient groups, regardless of the development of Grade 3/ 4 ALT/AST elevation, the large majority of patients did not experience a clinical hepatic SAE.

\section{Discussion and Conclusions}

The goal of cART is to achieve durable HIV suppression and sustained improvements in immune function by the delivery of an ARV regimen that also provides adequate safety and tolerability. Adverse events are associated with all ARV agents and may influence decisions to switch or discontinue therapy. Since some individuals may be predisposed to ARV therapy-associated AEs, safety considerations should take into account underlying conditions, concomitant medications and history of drug intolerance [11]. All ARV drugs have been associated with development of Grade 3/4 ALT/AST elevations [11,32]; and these events are more frequent in patients with $\mathrm{HBV} / \mathrm{HCV}$ coinfection or increased baseline hepatic transaminase lev- 


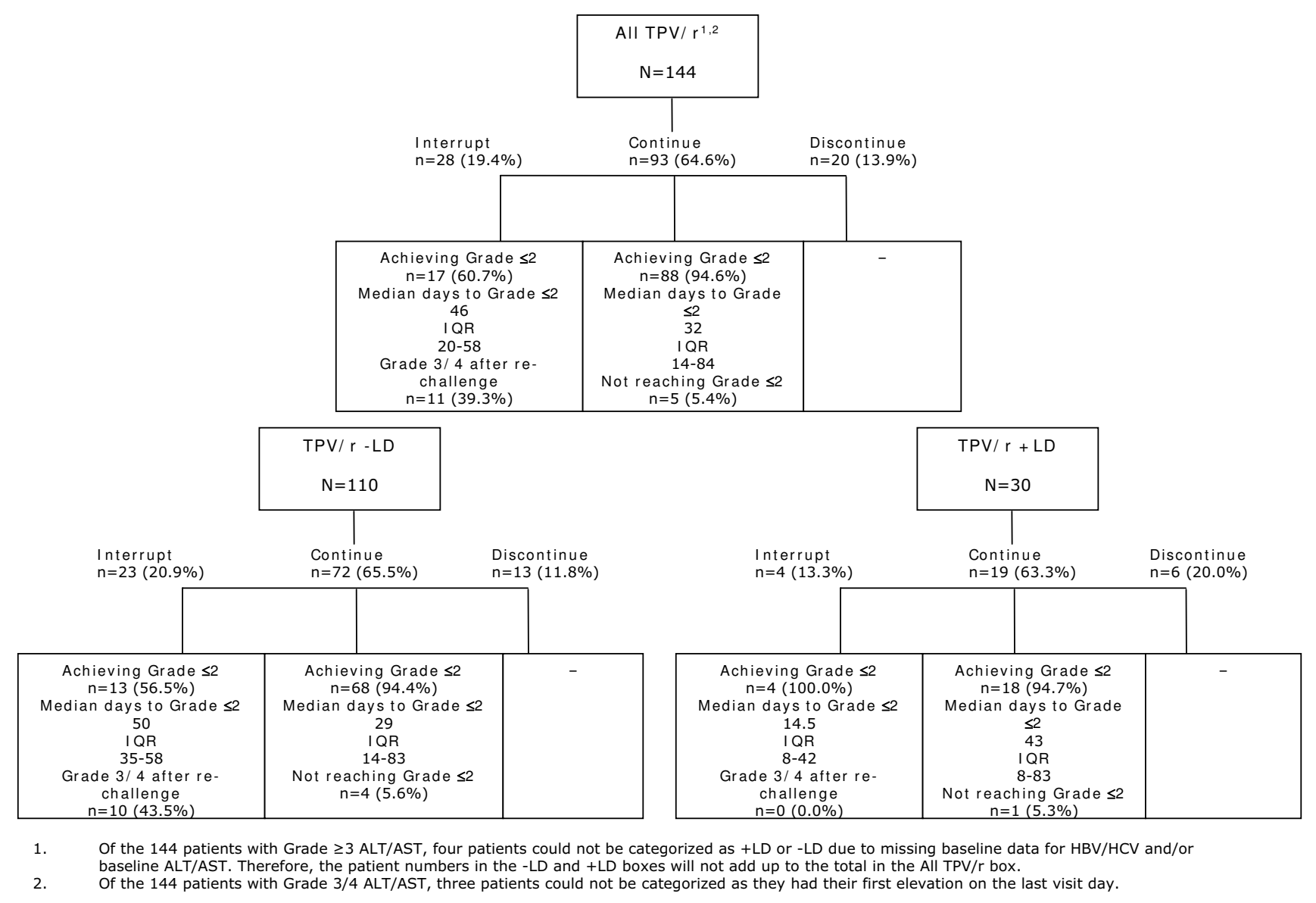

\section{Figure 2}

Actions and outcomes of Grade 3/4 ALT/AST elevations among TPV/r recipients. TPV/r +LD patients $=$ TPV/r patients co-infected with HBV/HCV or with baseline ALT/AST DAIDS $>1$. TPV/r -LD patients = TPV/r patients not co-infected with HBV/HCV and with baseline ALT/AST DAIDS I. +LD = patients with underlying liver disease (baseline evidence of active $\mathrm{HBV} / \mathrm{HCV}$ infection or ALT/AST DAIDS $>\mathrm{I}$ ); -LD = patients with no apparent liver disease (absence of active HBV/HCV infection and ALT/AST Grade $\leq \mathrm{I}$ ); ALT = alanine aminotransferase; AST = aspartate aminotransferase; HBV = hepatitis B virus; $\mathrm{HCV}=$ hepatitis $\mathrm{C}$ virus; $\mathrm{IQR}=$ interquartile range; $\mathrm{TPV} / \mathrm{r}=$ ritonavir-boosted tipranavir.

els [11,32-34]. However, the cause of liver enzyme elevations is often unclear, particularly among HIV-infected individuals with advanced disease. Further, in some asymptomatic individuals, elevated transaminases may resolve spontaneously despite continued drug treatment [35]. This may reflect an adaptive host response as seen with some drugs causing hepatotoxicity $[36,37]$.

The development of DAIDS Grade 3/4 transaminase elevations and clinical hepatic SAEs in treatment-experienced HIV-positive patients receiving ARV therapy with $\mathrm{TPV} / \mathrm{r}$ 500/200 mg BID varies according to baseline factors that define -LD and +LD patients. The K-M cumulative risk estimate of patients with DAIDS Grade 3/4 elevations by
Week 96 was lower among the -LD patients (12.5\%), compared to the $+\mathrm{LD}$ patients $(20.6 \%)$.

The large majority of TPV/r patients who developed DAIDS Grade 3/4 ALT/AST elevations remained asymptomatic and did not develop clinical signs or symptoms of liver-related SAEs. Among -LD patients, the cumulative risk of experiencing a hepatic SAE was low (1.0\%) through 96 weeks of treatment, with most occurring in the first 6 months. As expected, +LD patients tended to have a greater risk of developing a hepatic SAE than -LD patients.

Per protocol, any patient experiencing DAIDS 4 toxicity, according to the GCP and ICH guidelines, was to be dis- 
Table 4: Occurrence of DAIDS Grade 3/4 ALT/AST and hepatic serious adverse events (SAEs) among -LD and +LD TPV/r 500/ $200 \mathrm{mg}$ BID patients

\begin{tabular}{ccccc}
\hline & -LD patients & \multicolumn{2}{l}{ +LD patients } \\
\hline ALT/AST DAIDS $>\mathbf{2 2}^{2}$ & \multicolumn{2}{l}{ Hepatic SAE } & \multicolumn{2}{l}{ Hepatic SAE } \\
\hline & Yes & No & Yes & No \\
\hline Yes & 3 & 107 & I & 29 \\
\hline No & 6 & 970 & 4 & 141 \\
\hline
\end{tabular}

'Thirty-two patients had missing information and were unable to be classified into one of the two risk groups; none of these patients experienced a hepatic SAE.

$+\mathrm{LD}=$ patients with underlying liver disease (baseline evidence of active HBV/HCV infection or ALT/AST Grade >I); -LD = patients with no apparent liver disease (absence of active $\mathrm{HBV} / \mathrm{HCV}$ infection and ALT/AST Grade $\leq$ I); ALT = alanine aminotransferase; $A S T=$ aspartate aminotransferase; DAIDS = Division of AIDS; HBV = hepatitis $B$ virus; $\mathrm{HCV}=$ hepatitis $C$ virus; $T P V / r=$ ritonavir-boosted tipranavir

2ALT/AST on treatment DAIDS Grade 3/4

continued from treatment. However, the patient population consisted of patients with advanced stage of HIV disease, presenting with opportunistic infections and the $\mathrm{TPV} / \mathrm{r}$ was their last option for treatment. These compassionate trial designs were developed and approved by the regulatory authorities and key opinion leaders and the continued treatment despite toxicity was a decision between the principal investigator and the patient on a case by case basis with very close patient monitoring. Consequently, many patients who developed DAIDS Grade 3/ 4 ALT/AST elevations on TPV/r therapy were able to continue treatment uninterrupted or resume treatment after temporary discontinuation, with transaminase levels returning to DAIDS Grade 2 or less during the treatment period. Interestingly, changes in serum ALT/AST transaminase levels associated with TPV/r treatment were not typically predictive of the development of clinical hepatic SAEs. This lack of association of serum ALT/AST levels and clinical events has been observed with other medications. For example, at an FDA-AASLD-PhRMA (Food and Drug Administration-American Association for the Study of Liver Diseases-Pharmaceutical Research and Manufacturers of America) meeting on drug hepatotoxicity [36], it was pointed out that some drugs, such as isoniazid, are associated with a high risk of substantial transaminase elevations. However, these transaminase elevations are not strongly predictive of clinical hepatic events (low PPV) and clinical monitoring has become increasingly more important than laboratory monitoring. Nevertheless, transaminase elevations are a marker for hepatocellular liver injury so, despite poor PPV, increased transaminase monitoring is likely to be of value in $+\mathrm{LD}$ patients in whom the clinical significance of hepatic injury may be greater.

Multivariate analyses revealed hepatitis co-infection and baseline liver impairment being independently associated with increased risk of severe liver transaminase elevations. Others have also identified chronic viral hepatitis and abnormal baseline liver enzyme levels as risk factors for increased risk of hepatotoxicity with other ARV regimens $[9,11,16,20,21,23,35,38-41]$. The association between $\mathrm{CD}^{+}>200$ cells $/ \mathrm{mm}^{3}$ at baseline and the subsequent risk of developing DAIDS 3/4 TE while on treatment may have resulted from a bias. It is possible that the "healthier" patients with higher $\mathrm{CD} 4{ }^{+}$cell count at baseline remained on study longer than the sicker patients and therefore had more transaminase testing performed, and thus increasing the possibility of detection of elevated transaminases. In our study, patients with $\mathrm{CD}^{+}$counts $>200 \mathrm{cell} / \mathrm{mm}^{3}$ remained in the study nearly 3.5 months longer than patients with counts at or below 200 cells $/ \mathrm{mm}^{3}$. The overall drug exposure difference is more pronounced when one considers treatment regimen; on the average TPV/r patients with $\mathrm{CD}^{+}$counts above 200 remained in the study 8 month longer than their CPI/r treated counterparts (data not shown).

Inherent in many clinical trials are limitations in capturing all of the necessary risk factors associated with the outcome being evaluated. Although safety of patients was of paramount importance, the focus of these trials was on efficacy in a cohort of patients with highly advanced HIV disease and limited treatment options. As such, baseline information on known risk factors for hepatotoxicity, such as allergic predisposition, severe hepatic steatosis or hepatic cirrhosis [8] was obtained only through investigator and/or patient self-reporting; and therefore may have led to an underestimation of the presence of pre-existent liver disease, and hence an underestimation of the contribution of these to observed serious hepatic events associated with tipranavir in patients. In these trials, the baseline prevalence of any reported hepatic steatosis, cirrhosis or drug hypersensitivity was too low, $0.5 \%$ (6/ $1299), 0.2 \%$ (2/1299) and $1.9 \%$ (25/1299), respectively, to warrant evaluation of these important risk factors.

Another limitation to these analyses, found in HIV clinical studies in general, is the predominance of male patients. In the tipranavir Phase IIb and III clinical trials, only $10 \%$ of all patients were females, possibly limiting our ability to fully examine the role of gender on our study results and conclusions. While some researchers have shown a differential incidence of drug-related adverse events [42$44]$ based on gender, these results have not been confirmed by others $[45,46]$. 
In conclusion, TPV/r-based cART was associated with DAIDS Grade 3/4 ALT/AST elevations in treatment-experienced, HIV-1-infected patients with HIV advanced disease and limited treatment options; patients with elevated baseline ALT and/or AST levels and/or chronic viral hepatitis had the greatest risk. Of note, most of these transaminase elevations were not associated with clinically significant events; most patients with elevated transaminases experienced resolution without permanent discontinuation of TPV/r. Furthermore, Grade 3/4 ALT/AST elevations were not predictive of subsequent clinical hepatic events because the majority of patients remained asymptomatic. In this highly ARV-experienced patient population, the risk of DAIDS Grade 3/4 ALT/AST elevations should be balanced with the potential benefit of treatment with TPV/r in individuals for whom this represents an active ARV drug. Importantly, TPV/r demonstrated significantly greater virologic response than $\mathrm{CPI} / \mathrm{r}$ in the RESIST trials, which enrolled more than 1400 treatment-experienced patients $[6,30]$. Further follow-up of these trials has indicated that TPV/r patients who achieve virologic suppression continue to do well through 156 weeks of treatment [31].

For HIV-positive patients being treated with TPV/r, healthcare providers should monitor patients for the emergence of clinically significant hepatic events. Appropriate laboratory and clinical monitoring should be conducted prior to initiating therapy with $\mathrm{TPV} / \mathrm{r}$, and throughout treatment. Increased frequency of monitoring should be considered when TPV/r is administered to patients with elevated baseline ALT/AST levels, or active HBV/HCV coinfection, as these patients may be at increased risk for developing further transaminase elevations.

\section{Competing interests}

JM, PAR, MR and JOS are employees of Boehringer Ingelheim, the manufacturer of APTIVUS ${ }^{\circledast}$ (tipranavir). The authors declare that they have no other competing interests.

\section{Authors' contributions}

All authors participated in the data analysis and interpretation of results, as well as in revising the final manuscript.

\section{Acknowledgements}

All the studies discussed in this manuscript were supported by funding from Boehringer Ingelheim Pharmaceuticals, Inc.

\section{References}

I. Mocroft A, Vella S, Benfield TL, Chiesi A, Miller V, Gargalianos P, et al.: Changing patterns of mortality across Europe in patients infected with HIV-I. Lancet I998, 9 I 42: I725-I730.

2. Palella FJ Jr, Delaney KM, Moorman AC, Loveless MO, Fuhrer J, Satten GA, et al:: Declining morbidity and mortality among patients with advanced human immunodeficiency virus infection. HIV Outpatient Study Investigators. N Engl J Med 1998, 338:853-860.
3. Vittinghoff E, Scheer S, O'Malley P, Colfax G, Holmberg SD, Buchbinder SP: Combination antiretroviral therapy and recent declines in AIDS incidence and mortality. J Infect Dis 1999 , I79(3):7|7-720.

4. Drake JW: Rates of spontaneous mutation among RNA viruses. Proc Natl Acad Sci USA 1993, 90:417|-4I75.

5. Cooper $D$, Hall $D$, Jayaweera $D$, et al.: Baseline phenotypic susceptibility to tipranavir/ritonavir is retained in isolates from patients with multiple protease inhibitor experience (BI I I 82.52). [abstract 596]. Presented at: 10th Conference on Retroviruses and Opportunistic Infections; Boston 2003.

6. Hicks CB, Cahn P, Cooper DA, Walmsley SL, Katlama C, Clotet B, et al.: Durable efficacy of tipranavir-ritonavir in combination with an optimised background regimen of antiretroviral drugs for treatment-experienced HIV-I-infected patients at 48 weeks in the Randomized Evaluation of Strategic Intervention in multi-drug reSistant patients with Tipranavir (RESIST) studies: an analysis of combined data from two randomised open-label trials. Lancet 2006, 368:466-475.

7. Becker S: Liver Toxicity in Epidemiological Cohorts. Clinical Infectious Diseases 2004, 38:S49-S55.

8. Nunez M: Hepatotoxicity of antiretrovirals: incidence, mechanisms and management. J Hepatol 2006, 44:SI32-SI39.

9. Sulkowski MS, Mehta SH, Chaisson RE, Thomas DL, Moore RD: Hepatotoxicity associated with protease inhibitor-based antiretroviral regimens with or without concurrent ritonavir. AIDS 2004, 18:2277-2284.

10. Rodriguez-Rosado R, Garcia-Samaniego J, Soriano V: Hepatotoxicity after introduction of highly active antiretroviral therapy. [Letter]. AIDS 1998, I 2:1256.

II. Luzuriaga K, McManus M, Mofenson L, Britto P, Graham B, Sullivan JL: A trial of three antiretroviral regimens in HIV-I-infected children. N Engl J Med 2004, 350:247I-2480.

12. Crum NF, Riffenburgh RH, Wegner S, Agan BK, Tasker SA, Spooner $\mathrm{KM}$, et al.: Comparisons of causes of death and mortality rates among HIV-infected persons: analysis of the pre-, early, and late HAART (highly active antiretroviral therapy) eras. J Acquir Immune Defic Syndr 2006, 4I: 194-200.

13. Soriano V, Garcia-Samaniego J, Rodriguez-Rosado R, Gonzalez J, Pedreira J: Hepatitis C and HIV infection: biological, clinical, and therapeutic implications. J Hepatol 1999, 31 (Suppl I): I19-123.

14. Winnock M, Salmon-Ceron D, Dabis F, Chene G: Interaction between HIV-I and HCV infections: towards a new entity? J Antimicrob Chemother 2004, 53:936-946.

15. Schiavini M, Angeli E, Mainini A, Zerbi P, Duca PG, Gubertini G, et al:: Risk factors for fibrosis progression in HIV/HCV coinfected patients from a retrospective analysis of liver biopsies in 1985-2002. HIV Medicine 2006, 7:33I-337.

16. Sulkowski MS, Thomas DL, Chaisson RE, Moore RD: Hepatotoxicity associated with antiretroviral therapy in adults infected with human immunodeficiency virus and the role of hepatitis C or B virus infection. JAMA 2000, 283:74-80.

17. Sauleda S, Martorell M, Esteban JI, Tural C, Ruiz I, Puig L, et al.: Hepatotoxicity of antiretroviral drugs in HIV HCV patients with congenital coagulopathies followed at an Haemophilia Unit during a decade. Haemophilia 2006, I 2:228-236.

18. DAIDS: National Institutes of Health, Division of AIDS Table for Grading the Severity of Adult and Pediatric Adverse Events, version I.0. 2004 [http://www3.niaid.nih.gov/LabsAn dResources/resources/DAIDSClinRsrch/PDF/DAIDSAEGradingTa ble.pdf].

19. Sulkowski M, Rockstroh J, Soriana V, Stern J, Mikl J: Clinical course of increased LFTs and hepatic events associated with ritonavir (RTV)-boosted tipranavir (TPV/r) based therapy in the RESIST trials [abstract H-1 899]. Presented at: Interscience Conference on Antimicrobial Agents and Chemotherapy; San Francisco 2006.

20. Torti C, Lapadula G, Casari S, Puoti M, Nelson M, Quiros-Roldan E, et al.: Incidence and risk factors for liver enzyme elevation during highly active antiretroviral therapy in HIV-HCV coinfected patients: results from the Italian EPOKA-MASTER Cohort. BMC Infectious Diseases 2005, 5: I- 10.

21. Bonfanti P, Landonio S, Ricci E, Martinelli C, Fortuna P, Faggion I, et al.: Risk Factors for Hepatotoxicity in Patients Treated With Highly Active Antiretroviral Therapy. [Letter]. JAIDS Journal of Acquired Immune Deficiency Syndromes 200I, 27:3I6-3I8. 
22. Becker S: Liver toxicity in epidemiological cohorts. Clin Infect Dis 2004, 38:S49-S55.

23. Gisolf EH, Dreezen C, Danner SA, Weel JL, Weverling GJ: Risk factors for hepatotoxicity in HIV-I-infected patients receiving ritonavir and saquinavir with or without stavudine. Prometheus Study Group. Clin Infect Dis 2000, 31: I234-1239.

24. Servin-Abad L, Molina E, Baracco G, Arosemena L, Regev A, Jeffers L, et al.: Liver enzymes elevation after HAART in HIV-HCV coinfection. J Viral Hepat 2005, I 2:429-434

25. Gathe JC Jr, Pierone G, Piliero P, Arasteh K, Rubio R, Lalonde RG, et al.: Efficacy and safety of three doses of tipranavir boosted with ritonavir in treatment-experienced HIV type-I infected patients. AIDS Res Hum Retroviruses 2007, 23:216-223.

26. Leith J, Walmsley S, Katlama C, et al.: Pharmacokinetics and safety of tipranavir/ritonavir (TPV/r) alone or in combination with saquinavir (SQV), amprenavir (APV), or lopinavir (LPV): interim analysis of BII I 82.5 I [abstract 5.I]. Presented at: 5th International Workshop on Clinical Pharmacology of HIV Therapy; Rome 2004

27. Cahn P, Villacian J, Lazzarin A, Katlama C, Grinsztejn B, Arasteh K, et al.: Ritonavir-boosted tipranavir demonstrates superior efficacy to ritonavir-boosted protease inhibitors in treatmentexperienced HIV-infected patients: 24-week results of the RESIST-2 trial. Clin Infect Dis 2006, 43:1347-I356.

28. Gathe J, Cooper DA, Farthing C, Jayaweera D, Norris D, Pierone G $J$, et al.: Efficacy of the protease inhibitors tipranavir plus ritonavir in treatment-experienced patients: 24-week analysis from the RESIST-I trial. Clin Infect Dis 2006, 43: I337-I346.

29. Pierone G, Drulak M, Arastéh K, et al.: A long-term open-labe rollover trial assessing the safety and tolerability of combination tipranavir and ritonavir (TPV/r) use in HIV-I-infected patients [poster WePe6.2C05]. Presented at: 3rd International AIDS Conference; Rio de Janeiro 2005.

30. Farthing C, Ward D, Hicks C, et al: Tipranavir/r demonstrates superior and durable treatment response compared with comparator $\mathrm{PI} / \mathrm{r}$ in highly treatment experienced patients: Week 96 RESIST I and 2 results [abstract $\mathbf{H}-\mathbf{1 3 8 5}$ ]. Presented at: 46th Interscience Conference on Antimicrobial Agents and Chemotherapy; San Francisco :2006.

31. Hicks C, Cahn P, Jelaska A, Drulak M, Vinisko R: Tipranavir/r (TPV r) maintains long term virological suppression - Week 156 results of RESIST [poster P7.3/25]. Presented at: I I th European AIDS Conference; Madrid 2007.

32. Orenstein R, Tsogas N: Looking Beyond Highly Active Antiretroviral Therapy: Drug-Related Hepatotoxicity in Patients with Human Immunodeficiency Virus Infection. Pharmacotherapy 2002, 22: |468-| 478 .

33. Sulkowski MS, Thomas DL, Mehta SH, Chaisson RE, Moore RD: Hepatotoxicity associated with nevirapine or efavirenz-containing antiretroviral therapy: role of hepatitis $\mathbf{C}$ and $B$ infections. Hepatology 2002, 35:182-189.

34. Sulkowski MS: Drug Induced Liver Injury Associated with Antiretroviral Therapy that Includes HIV I Protease Inhibitors. Clinical Infectious Diseases 2004, 38:590-S97.

35. den Brinker M, Wit FW, Wertheim-van Dillen PM, Jurriaans S, Weel J, van $L R$, et al: Hepatitis $B$ and $C$ virus co-infection and the risk for hepatotoxicity of highly active antiretroviral therapy in HIV-I infection. AIDS 2000, 14:2895-2902.

36. John R, Senior M, AASLD-FDA-NIH-PhRMA Hepatotoxicity Steering Group Meeting 2006 Presentations: Adaptation to Liver Injury Tacrine, Isoniazid, Ethanol, Experimental Drugs. 2006 [http:/ /www.fda.gov/downloads/Drugs/ScienceResearch/ResearchAreas/ ucm079327.pdf ].

37. Scherpbier HJ, Bekker V, van LF, Jurriaans S, Lange JM, Kuijpers TW: Long-term experience with combination antiretroviral therapy that contains nelfinavir for up to 7 years in a pediatric cohort. Pediatrics 2006, II 7:e528-e536.

38. Kress KD: Antiretroviral-associated Hepatotoxicity. Curr Infect Dis Rep 2005, 7:103-107.

39. Brau N, Bini El, Shahidi A, Aytaman A, Xiao P, Stancic S, et al.: Prevalence of hepatitis $C$ and coinfection with HIV among United States veterans in the New York City metropolitan area. Am J Gastroenterol 2002, 97:207|-2078.

40. Sherman KE, Rouster SD, Chung RT, Rajicic N: Hepatitis C Virus prevalence among patients infected with Human Immuno- deficiency Virus: a cross-sectional analysis of the US adult AIDS Clinical Trials Group. Clin Infect Dis 2002, 34:83I-837.

4I. Sulkowski MS, Moore RD, Mehta SH, Chaisson RE, Thomas DL: Hepatitis C and progression of HIV disease. JAMA 2002, 288: 199-206

42. Umeh OC, Currier JS: Sex differences in pharmacokinetics and toxicity of antiretroviral therapy. Expert Opin Drug Metab Toxicol 2006, 2:273-283.

43. Guitton E, Montastruc JL, Lapeyre-Mestre M: Influence of HCV or HBV coinfection on adverse drug reactions to antiretroviral drugs in HIV patients. Eur J Clin Pharmacol 2006, 62:243-249.

44. Wester CW, Okezie OA, Thomas AM, Bussmann H, Moyo S, Muzenda $T$, et al.: Higher-than-expected rates of lactic acidosis among highly active antiretroviral therapy-treated women in Botswana: preliminary results from a large randomized clinical trial. J Acquir Immune Defic Syndr 2007, 46:3 I 8-322.

45. Mould DR, Zhang X, Nieforth K, Salgo M, Buss N, Patel IH: Population pharmacokinetics and exposure-response relationship of enfuvirtide in treatment-experienced human immunodeficiency virus type I-infected patients. Clin Pharmacol Ther 2005, 77:5I5-528.

46. Johnson MO, Charlebois E, Morin SF, Catz SL, Goldstein RB, Remien $\mathrm{RH}$, et al:: Perceived adverse effects of antiretroviral therapy. J Pain Symptom Manage 2005, 29:193-205.

\section{Pre-publication history}

The pre-publication history for this paper can be accessed here:

\section{http://www.biomedcentral.com/1471-2334/9/203/pre} pub
Publish with Bio Med Central and every scientist can read your work free of charge

"BioMed Central will be the most significant development for disseminating the results of biomedical research in our lifetime. "

Sir Paul Nurse, Cancer Research UK

Your research papers will be:

- available free of charge to the entire biomedical community

- peer reviewed and published immediately upon acceptance

- cited in PubMed and archived on PubMed Central

- yours - you keep the copyright
BiolMedcentral 